\title{
HIGHER $R$-DERIVATIONS OF SPECIAL SUBRINGS OF MATRIX RINGS
}

\author{
By \\ Andrzej NowickI
}

\section{Introduction.}

Let $R$ be a ring with identity and $P$ be a special subring of $M_{n}(R)$ ([7]), i.e. $P$ is of the form

$$
P=\left\{A \in M_{n}(R) ; A_{i j}=0 \text { for }(i, j) \oplus \rho\right\},
$$

where $\rho$ is a (reflexive and transitive) relation on the set $\{1,2, \cdots, n\}$, and $M_{n}(R)$ is the ring of $n \times n$ matrices over $R$.

In this paper we study the group $D_{s}^{R}(P)$ of all $R$-derivations of order $s([5]$, [8]-[11]) of $P$. We prove Theorem 5.3 that every element $d \in D_{s}^{R}(P)$ has a unique representation of the form $d=d^{(1)} * d^{(2)}$, where $d^{(1)}$ is an inner derivation in $D_{s}^{R}(P)([8])$, and $d^{(2)}$ is an element of a certain abelian subgroup of $D_{s}^{R}(P)$ whose simple description is given in Section 3 (by $*$ we denote the multiplication in the group $D_{s}^{R}(P)$ ). This theorem plays a basic role in our further considerations.

Moreover, in Section 4, we give some necessary and suffiecient conditions for a ring $P$ to have all $R$-derivations (all derivations) of order $s$ of $P$ to be inner.

In Sections 7,8,9 we investigate $s^{\prime}$-integrable $R$-derivations of order $s$ (where $\left.s<s^{\prime}\right)$ i.e. such $R$-derivations of order $s$ which can be extended to $R$-derivations of order $s^{\prime}$ (comp. [4]). We show in Example 7.4 that, in general, there are non-integrable $R$-derivations of $P$. We prove Theorem 9.6 that if the homology group $H_{1}(\Gamma)$ of the simplicial complex $\Gamma$ of the relation $\rho$ (Section 2) is free abelian, then every usual $R$-derivation is 3-integrable, and if, in addition, $H_{2}(\Gamma)$ $=0$ then every $R$-derivation of order $s$ is $s^{\prime}$-integrable for any $s<s^{\prime}$ Theorem 8.6).

At the end of this paper, we formulate three open problems.

\section{Preliminaries.}

Throughout this paper $R$ is a ring with identity, $n$ is a fixed natural number and $\rho$ is a reflexive and transitive relation on the set $I_{n}=\{1,2, \cdots, n\}$. 
We denote by $M_{n}(R)$ the ring of $n \times n$ matrices over $R$ and by $Z(R)$ the center of $R$.

Moreover, we use the following conventions:

$S=$ a segment of $N=\{0,1, \cdots\}$, that is, $S=N$ or $S=\{0,1, \cdots, k\}$ for some integer $k \geqq 0$

$s=\sup (S) \leqq \infty$,

$A_{i j}=i j$-coefficient of a matrix $A$,

$E^{i j}=$ the element of the standard basis of $M_{n}(R)$,

$\bar{r}=$ the diagonal matrix whose all coefficients on the diagonal are equal to $r \in R$,

$M_{n}(R)_{\rho}=$ the set $\left\{A \in M_{n}(R) ; A_{i j}=0\right.$ for $\left.(i, j) \notin \rho\right\}$.

It is clear, that $M_{n}(R)_{\rho}$ is a subring of $M_{n}(R)$. (Conversely, if $\sigma$ is a reflexive relation on $I_{n}$ and $M_{n}(R)_{\sigma}$ is a subring of $M_{n}(R)$, then $\sigma$ is transitive). We say that the subring $P=M_{n}(R)_{\rho}$ of $M_{n}(R)$ is special with the relation $\rho$.

Let $P$ be an arbitrary ring with identity. A sequence $d=\left(d_{m}\right)_{m \in S}$ of mappings $d_{m}: P \rightarrow P$ is called a derivation of order $s$ of $P$ (see [5], [8], [9], [10], [11]) if the sollowing properties are satisfied:

(1) $d_{m}(a+b)=d_{m}(a)+d_{m}(b)$,

(2) $\quad d_{m}(a b)=\sum_{i+j=m} d_{i}(a) d_{j}(b)$,

(3) $d_{0}(a)=a$,

for all $m \in S$ and $a, b \in P$.

The set $D_{s}(P)$ of all derivations of order $s$ of $P$ is a group under the multiplication $*$ defined by the formula

$$
\left(d * d^{\prime}\right)_{m}=\sum_{i+j=m} d_{i} \circ d_{j}^{\prime},
$$

wehre $d, d^{\prime} \in D_{s}(P)$ and $m \in S([9],[10],[4])$.

If $a \in P$ and $k \in S \backslash\{0\}$ then by $[a, k]$ we denote the element of $D_{s}(P)$ defined by

for $m \in S, x \in P([8])$.

$$
[a, k]_{m}(x)= \begin{cases}x, & \text { if } m=0, \\ 0, & \text { if } k \nmid m, \\ a^{r} x-a^{r-1} x a, & \text { if } \quad m=k r>0,\end{cases}
$$

If $\underline{a}=\left(a_{m}\right)_{m \in S \backslash 0\}}$ is a sequence of elements of $P$ then by $\Delta(\underline{a})$ we denote the inner derivation of order $s$ of $P$ with respect to $\underline{a}([[8])$, i.e., $\Delta(\underline{a})$ is a derivation of order $s$ of $P$ such that 


$$
\Delta(\underline{a})_{m}=\left(\left[a_{1}, 1\right] * \cdots *\left[a_{m}, m\right]\right)_{m}
$$

for all $m \in S$. The set of inner derivations of order $s$ of $P$, denoted by $I D_{s}(P)$, is a normal subgroup of $D_{s}(P)$ ([8] Corollary 3.3).

Recall that the usual derivation of $P$ is an additive mapping $\delta: P \rightarrow P$ such that $\delta(a b)=\delta(a) b+a \delta(b)$, for all $a, b \in P$.

The set of usual derivations of $P$ corresponds bijectively to the set $D_{1}(P)$, namely if $d \in D_{s}(P)$ then $d_{1}$ is an usual derivation of $P$.

We now assume that $P$ is a special subring of $M_{n}(R)$ with the relation $\rho$.

Observe that we can extend every derivation of order $s$ of $R$ to a derivation of order $s$ of $P$.

Indeed, if $\delta \in D_{s}(R)$ then the sequence $d=\left(d_{m}\right)_{m \in S}$ of mappings $d_{m}: P \rightarrow P$ defined by $d_{m}(A)_{i j}=\delta_{m}\left(A_{i j}\right)$ (for $A \in P, \quad m \in S$ ) is a derivation of order $s$ of $P$ such that $d_{m}(\bar{r})=\overline{\delta_{m}(r)}$ for any $r \in R, m \in S$.

Look also on a generalization of the above fact.

EXAMPLE 2.1. Let $\bar{\rho}$ be the smallest equivalence relation on $I_{n}$ containing $\rho, T$ a fixed set of representatives of equivalence classes of $\bar{\rho}$, and $v: I_{n} \rightarrow T$ the mapping defined by :

$$
v(p)=t \quad \text { iff } \quad p \bar{\rho} t
$$

Moreover, let $\underline{d}=\left(d^{(t)}\right)_{t \in T}$ be a sequence of elements of $D_{s}(R)$. Consider the sequence $\Theta(\underline{d})=\left(d_{m}\right)_{m \in S}$ of mappings from $P$ to $P$ defined as follows

$$
d_{m}(A)_{i j}=d_{m}^{(v(i))}\left(A_{i j}\right)
$$

for all $m \in S, A \in P$.

It is easy to verify that $\Theta(\underline{d})$ belongs to $D_{s}(P)$.

If a derivation $d \in D_{s}(P)$ satisfies following equivalent two conditions :

(4) $d_{m}(\bar{r} A)=\bar{r} d_{m}(A)$ for all $m \in S, r \in R, A \in P$,

(5) $d_{m}(\bar{r})=0$ for all $m \in S \backslash\{0\}, r \in R$,

then $d$ is called $R$-derivation of order $s$ of $P$, and the set of all such derivations is denoted by $D_{s}^{R}(P)$.

We define similarly an usual $R$-derivation, an inner $R$-derivation and the set $I D_{s}^{S}(P)$. It is clear, that $D_{s}^{R}(P)$ is a subgroup of $D_{s}(P)$, and (by [8] Corollary 3.3) $I D_{s}^{R}(P)$ is a normal subgroup of $D_{s}^{R}(P)$. An inner derivation $\Delta(\underline{A})$, where $\underline{A}=\left(A^{(m)}\right)_{m \in S \backslash(0\}}$ is a sequence of matrices of $P$, belongs to $I D_{s}^{R}(P)$ iff $A^{(m)} \in$ $M_{n}(Z(R))$ for any $m$. 
LEMMA 2.2. If $d \in D_{s}^{R}(P)$ then $d_{m}\left(E^{p q}\right)_{i j} \in Z(R)$ for any $m \in S$ and all $i, j, p, q$ $\in I_{n}$ such that poq.

PROOF. Let $r \in R$. Sinวe $\bar{r} E^{p q}-E^{p q} \bar{r}=0$ then

$$
\begin{aligned}
0 & =d_{m}\left(\bar{r} E^{p q}-E^{p q} \bar{r}\right)_{i j} \\
& =\sum_{u+v=m}\left(d_{u}(\bar{r}) d_{v}\left(E^{p q}\right)-d_{u}\left(E^{p q}\right) d_{v}(\bar{r})\right)_{i j} \\
& =\left(\bar{r} d_{m}\left(E^{p q}\right)-d_{m}\left(E^{p q}\right) \bar{r}\right)_{i j} \\
& r d_{m}\left(E^{p q}\right)_{i j}-d_{m}\left(E^{p q}\right)_{i j} r
\end{aligned}
$$

Usual derivations and usual $R$-derivations of $P$ are investigated in [6], [1], [2], [7]. In this paper (Section 5) we give a description of the group $D_{\boldsymbol{s}}^{R}(P)$.

Let $s<\infty$, and $S^{\prime}$ be a segment of $N$ such that $S \subsetneq S^{\prime}$. We say (comp. [4]) that an $R$-derivation $d \in D_{s}^{R}(P)$ is $s^{\prime}$-integrable (where $s^{\prime}=\sup \left(S^{\prime}\right) \leqq \infty$ ) if there exists an $R$-derivation $d^{\prime} \in D_{s^{\prime}}^{R}(P)$ such that $d_{m}^{\prime}=d_{m}$ for all $m \in S$. We will study such derivations in Sections 7, 8, 9.

Now we will define the graph $\Gamma$ of the relation $\rho$. Let $\sim$ be the equivalence relation on $I_{n}$ defined by :

$$
x \sim y \text { iff } x \rho y \text { and } y \rho x .
$$

Denote by $[x]$ the equivalence class of $x \in I_{n}$ with respect to $\sim$, and let $I_{n}^{\prime}$ be the set of all equivalence classes. We define a relation $\rho^{\prime}$ of partial order on $I_{n}$ as follows:

$$
[x] \rho^{\prime}[y] \text { iff } x \rho y \text {. }
$$

We will denote the pair $\left(I_{n}^{\prime}, \rho^{\prime}\right)$ by $\Gamma$ ( or $\left.\Gamma(\rho)\right)$ and calle it the graph of $\rho$. Elements of $I_{n}^{\prime}$ we calle vertices of $\Gamma$ and pairs $(a, b)$, where $a \rho^{\prime} b$ and $a \neq b$. arrows of $\Gamma$.

Let us imbed the set of the vertices of $\Gamma$ in an Euclidean space of a sufficiently high dimension so that the vertices will be linearly independent.

If $a_{0}, a_{1}, \cdots, a_{k}$ are elements of $I_{n}^{\prime}$ such that $a_{i} \rho^{\prime} a_{i+1}$ and $a_{i} \neq a_{i+1}$ for $i=$ $0,1, \cdots, k-1$, then by $\left(a_{0}, a_{1}, \cdots, a_{k}\right)$ we denote the $k$-dimensional simplex with vertices $a_{0}, \cdots, a_{k}$ ([3]). The union of all $0,1,2$ or 3 -dimensional such simplicies we will denote also by $\Gamma$. Therefore, $\Gamma$ is a simplicial complex of dimension $\leqq 3$.

Let $C_{k}(\Gamma)$, for $k=0,1,2,3$, be the free abelian group whose free generetors are $k$-dimensional simplicies of the complex $\Gamma$. We have the following standard complex of abelian groups : 


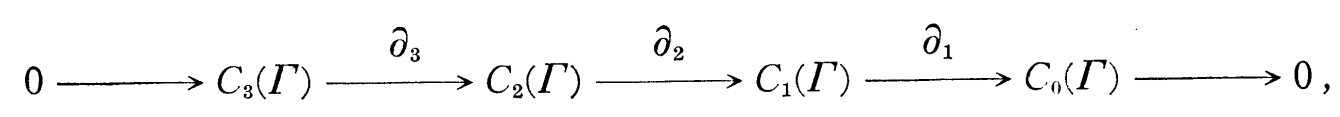

where

$$
\begin{aligned}
& \partial_{1}(a, b)=(b)-(a), \\
& \partial_{2}(a, b, c)=(b, c)-(a, c)+(a, b), \\
& \partial_{3}(a, b, c, d)=(b, c, d)-(a, c, d)+(a, b, d)-(a, b, c) .
\end{aligned}
$$

Then $H_{1}(\Gamma)=\operatorname{Ker} \partial_{1} / \operatorname{Im} \partial_{2}, H_{2}(\Gamma)=\operatorname{Ker} \partial_{2} / \operatorname{Im} \partial_{3}$ and (by the Künneth formulas)

$$
H^{1}(\Gamma, G)=\operatorname{Hom}\left(H_{1}(\Gamma), G\right)
$$

for an arbitrary abelian group $G$ (see [3]).

In the sequel $P$ denotes a special subring of $M_{n}(R)$ with the relation $\rho$.

\section{Transitive mappings.}

Recall from [7] that a mapping $\varphi: \rho \rightarrow Z(R)$ is called transitive if $\varphi(p, r)=$ $\varphi(p, q)+\varphi(q, r)$ for $p \rho q, q \rho r$. In this paper such mappings will be called usual transitive mappings from $\rho$ to $R$.

DeFinition 3.1. A sequence $f=\left(f_{m}\right)_{m \in S}$ of mappings $f_{m}: \rho \rightarrow Z(R)$ is called a transitive mapping of order $s$ from $\rho$ to $R$ if the following properties are satisfied :

(a) $f_{0}(p, q)=1$ for all $p \rho q$,

(b) $f_{m}(p, r)=\sum_{i+j=m} f_{i}(p, q) f_{j}(q, r)$ for all $m \in S$ and $p \rho q \rho r$.

We denote by $T M_{s}(\rho, R)$ the set of transitive mappings of order $s$ from $\rho$ to $R$.

By the above definition it follows that if $f \in T M_{s}(\rho, R)$ then

$$
f_{1}(p, r)-f_{1}(p, q)-f_{1}(q, r)=0,
$$

i.e. $f_{1}$ is an usual transitive mapping from $\rho$ to $R$, and

$$
\begin{aligned}
& f_{2}(p, r)-f_{2}(p, q)-f_{2}(q, r)=f_{1}(p, q) f_{1}(q, r), \\
& f_{3}(p, r)-f_{3}(p, q)-f_{3}(q, r)=f_{1}(p, q) f_{2}(q, r)+f_{2}(p, q) f_{1}(q, r)
\end{aligned}
$$

for all $p \rho q \rho r$.

It is easy to prove

LEMmA 3.2. (1) $f_{m}(p, p)=0$, for all $p \in I_{n}, m \in S \backslash\{0\}$.

(2) If $p \rho q$ and $q \rho p$, and $f_{2}(p, q)=\cdots=f_{m}(p, q)=0$ for some $m \geqq 2$, then 
$f_{k}(p, q)=(-1)^{k} f_{1}(p, q)^{k}=f_{1}(q, p)^{k}$ for $k=0, \cdots, m$.

EXAMPLE 3.3. If $Q \subseteq R$ and $\varphi: \rho \rightarrow Z(R)$ is an usual transitive mapping then the sequence $\left(f_{m}\right)_{m \in S^{\prime}}$ where $f_{m}(p, q)=(m !)^{-1} \varphi(p, q)^{m}$, is a transitive mapping of order $s$ from $\rho$ to $R$.

EXAMPLE 3.4. Let

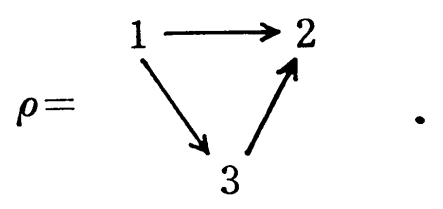

Put $f_{m}(1,2)=f_{m}(1,3)=1$ and $f_{m}(2,3)=0$ for all $m \in S \backslash\{0\}$. Then $f=\left(f_{m}\right)_{m \in S}$ belongs to $T M_{s}(\rho, R)$.

EXAMPLE 3.5. Let

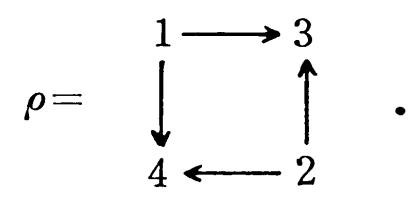

If $f_{m}$, for any $m \in S \backslash\{0\}$, is an arbitrary mapping from $\rho$ to $Z(R)$ then $\left(f_{m}\right)_{m \in S}$ is a transitive mapping of order $s$ from $\rho$ to $R$.

Let $f, g \in T M_{s}(\rho, R)$. Denote by $f * g$ the sequence $\left(h_{m}\right)_{m \in S}$ of mappings from $\rho$ to $Z(R)$ defined by

$$
h_{m}(p, q)=\sum_{i+j=m} f_{i}(p, q) g_{j}(p, q)
$$

for all $m \in S$ and $p \rho q$.

Then $f * g$ belongs to $T M_{s}(\rho, R)$ and it is easy to check that the set $T M_{s}(\rho, R)$, under the multiplication $*$, is an abelian group.

For every $f \in T M_{s}(\rho, R)$ we will denote by $\Delta^{f}$ the sequence $\left(\Delta_{m}^{f}\right)_{m \in S}$ of mappings $\Delta_{m}^{f}: P \rightarrow P$ defined by the following formula

$$
\Delta_{m}^{f}(A)_{p q}=f_{m}(p, q) A_{p q},
$$

for all $A \in P$ and $p \rho q$.

Then we have

LEMMA 3.6. The sequence $\Delta^{f}$ is an R-derivation of order $s$ of $P$.

Proof. Every $\Delta_{m}^{f}$ is obviously an $R$-additive mapping. Let $A, B \in P$ and 
$p \rho q$. Then

$$
\begin{aligned}
\left(\sum_{k=0}^{m} \Delta_{k}^{f}(A) \Delta_{m-k}^{f}(B)\right)_{p q} & =\sum_{k=0}^{m} \sum_{i=1}^{n} \Delta_{k}^{f}(A)_{p i} \Delta_{m-k}^{f}(B)_{i q} \\
& =\sum_{k=0}^{m} \sum_{i=1}^{n} f_{k}(p, i) f_{m-k}(i, q) A_{p i} B_{i q} \\
& =\sum_{i=1}^{n} f_{m}(p, q) A_{p i} B_{i q} \\
& =f_{m}(p, q)(A B)_{p q} \\
& =\Delta_{m}^{f}(A B)_{p q} .
\end{aligned}
$$

Therefore

$$
\Delta_{m}^{f}(A B)=\sum_{k=0}^{m} \Delta_{k}^{f}(A) \Delta_{m-k}^{f}(B),
$$

for all $m \in S$ and $A, B \in P$.

Proposition 3.7. The mapping $f \mapsto \Delta^{f}$ is a group monomorphism from $T M_{s}(\rho, R)$ to $D_{s}^{R}(P)$.

Proof. The condition $\Delta^{f * g}=\Delta^{f} * \Delta^{g}$ follows from definition of multiplications. Suppose now that $\Delta^{f}=\Delta^{g}$ for some $f, g \in T M_{s}(\rho, R)$. Then, for $p \rho q$ and $m \in S$, we have

$$
f_{m}(p, q)=\Delta_{m}^{f}\left(E^{p q}\right)_{p q}=\Delta_{m}^{g}\left(E^{p q}\right)_{p q}=g_{m}(p, q)
$$

i.e. $f=g$.

\section{Inner derivations.}

Recall from [7] that if $f$ is an usual transitive mapping from $\rho$ to $R$ then $f$ is called trivial iff there exists a mapping $\sigma: I_{n} \rightarrow Z(R)$ such that $f(p, q)=\sigma(p)-$ $\sigma(q)$ for all $p \rho q$. We say that the relation $\rho$ is regular over $R$ iff every usual transitive mapping from $\rho$ to $R$ is trivial.

Combining [8] Theorem 4.2 with results of the paper [7] we obtain the following two theorems

THEOREM 4.1. Let $P$ be a special subring of $M_{n}(R)$ with the relation $\rho$. The following conditions are equivalent:

(1) Every R-derivation of order $s$ of $P$ is inner,

(2) Every usual R-derivation of $P$ is inner,

(3) The relation $\rho$ is regular over $Z(R)$,

(4) The relation $\rho^{\prime}$ is regular over $Z(R)$, 
(5) $\quad H^{1}(\Gamma(\rho), Z(R))=0$.

THEOREM 4.2. Let $P$ be a special subring of $M_{n}(R)$ with the relation $\rho$. Denote by $w, w_{s}, u, u^{\prime}$ the following sentences:

$w=$ "Every usual derivation of $R$ is inner",

$w_{s}=$ "Every derivation of order $s$ of $R$ is inner",

$u=$ "The relation $\rho$ is regular over $Z(R)$ ",

$u^{\prime}=$ "The relation $\rho^{\prime}$ is regular over $Z(R)$ ".

Then the following conditions are equivalent:

(1) Every derivation of order $s$ of $P$ is inner,

(2) Every usual derivation of $P$ is inner.

(3) $w$ and $u$,

(4) $w_{s}$ and $u$,

(5) $w$ and $u^{\prime}$,

(6) $w_{s}$ and $u^{\prime}$,

(7) $w$ and $H^{1}(\Gamma(\rho), Z(R))=0$,

(8) $w_{s}$ and $H^{1}(\Gamma(\rho), Z(R))=0$.

EXAMPLE 4.3. If $P=M_{n}(R)_{\rho}$ where

a) $n \leqq 3$, or

b) the graph $\Gamma(\rho)$ is a tree, or

c) the graph $\Gamma(\rho)$ is a conne (i.e. there exists $b \in I_{n}$ such that $b \rho a$ or $a \rho b$ for any $\left.a \in I_{n}\right)$ in particular $P=M_{n}(R)$ or $P$ is the ring of triangular $n \times n$ matrices over $R$, or

d) the graph $\Gamma(\rho)$ is of the form

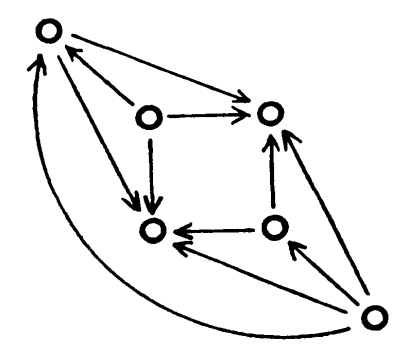

then every $R$-derivation (or every derivation, if every usual derivation of $R$ is inner) of order $s$ of $P$ is inner (see [7]]).

\section{The group $D_{s}^{R}(P)$.}

In this section we give a description of the group $D_{s}^{R}(P)$. 
We start from the following two lemmas.

Lemma 5.1. Let $d \in D_{s}^{R}(P), m \in S \backslash\{0\}$. Assume that $d_{k}\left(E^{q q}\right)_{p q}=0$ for $k=$ $1,2, \cdots, m$ and all $p \neq q$. Then

(i) $d_{k}\left(E^{p p}\right)_{p p}=0$ for $k=1,2, \cdots, m$ and any $p \in I_{n}$,

and

(ii) $d_{k}\left(E^{i j}\right)_{p q}=0$ for $k=1,2, \cdots, m$ and all $i \rho j, p \rho q$ such that $(p, q) \neq(i, j)$.

Proof. (by induction with respect to $m$ ). If $m=1$ then this lemma follows from [7] Lemma 3.1. Let $m>1$ and suppose that the conditions (i) and (ii) hold for any $k<m$. We show that then

(1) $d_{m}\left(E^{i j}\right)_{p q}=0$ for $i \neq p, j \neq q$,

(2) $d_{m}\left(E^{p p}\right)_{p p}=0$ for any $p \in I_{n}$,

(3) $d_{m}\left(E^{p p}\right)_{p_{j}}=0$ for $p \neq j$,

(4) $d_{m}\left(E^{p q}\right)_{i q}=0$ for $p \neq i$,

(5) $d_{m}\left(E^{p q}\right)_{p j}=0$ for $q \neq j$.

For example we verify (1) and (2). The proofs of the conditions (3)-(5) are similar.

(1) Let $i \neq p, j \neq q$, and $p \rho q, i \rho j$. Then

$$
\begin{aligned}
d_{m}\left(E^{i j}\right)_{p q} & =d_{m}\left(E^{i j} E^{j j}\right)_{p q} \\
& =\sum_{k+l=m}\left(d_{k}\left(E^{i j}\right) d_{l}\left(E^{j j}\right)\right)_{p q} \\
& =\sum_{k+l=m} \sum_{r} d_{k}\left(E^{i j}\right)_{p r} d_{l}\left(E^{j j}\right)_{r q} .
\end{aligned}
$$

Hence, by induction, we have

$$
\begin{aligned}
d_{m}\left(E^{i j}\right)_{p q} & =\sum_{r}\left(d_{0}\left(E^{i j}\right)_{p r} d_{m}\left(E^{j j}\right)_{r q}+d_{m}\left(E^{i j}\right)_{p r} d_{0}\left(E^{j j}\right)_{r q}\right) \\
& =\sum_{r}\left(0 d_{m}\left(E^{i j}\right)_{r q}+d_{m}\left(E^{i j}\right)_{p r} 0\right)=0 .
\end{aligned}
$$

(2) Let $p \in I_{n}$. Then

$$
\begin{aligned}
d_{m}\left(E^{p p}\right)_{p p} & =d\left(E^{p p} E^{p p}\right)_{p p} \\
& =\sum_{i+j=m}\left(d_{i}\left(E^{p p}\right) d_{j}\left(E^{p p}\right)\right)_{p p} \\
& =\sum_{i+j=m} \sum_{r} d_{i}\left(E^{p p}\right)_{p r} d_{j}\left(E^{p p}\right)_{r p} \\
& =\sum_{r}\left(d_{0}\left(E^{p p}\right)_{p r} d_{m}\left(E^{p p}\right)_{r p}+d_{m}\left(E^{p p}\right)_{p r} d_{0}\left(E^{p p}\right)_{r p}\right) \\
& =d_{m}\left(E^{p p}\right)_{p p}+d_{m}\left(E^{p p}\right)_{p p} .
\end{aligned}
$$


Hence $d_{m}\left(E^{p p}\right)_{p p}=0$.

LEMmA 5.2. Let $d \in D_{s}^{R}(P)$. Assume that $d_{m}\left(E^{q q}\right)_{p q}=0$ for all $m \in S \backslash\{0\}$ and all poq. Then the sequence $f=\left(f_{m}\right)_{m \in S}$ of mappings from $\rho$ to $R$ defined by $f_{m}(p, q)=d_{m}\left(E^{p q}\right)_{p q}$ for $p \rho q$ is a transitive mapping of order $s$ from $\rho$ to $R$.

Proof. Lemma 2.2 implies that $f_{m}(p, q) \in Z(R)$ for all $p \rho q$. Now let $p \rho q \rho r$, $m \in S$. By Lemma 5.1 we have

$$
\begin{aligned}
f_{m}(p, r) & =d_{m}\left(E^{p r}\right)_{p r}=d_{m}\left(E^{p q} E^{q r}\right)_{p r} \\
& =\left(\sum_{i+j=m} d_{i}\left(E^{p q}\right) d_{j}\left(E^{q r}\right)\right)_{p r} \\
& =\sum_{i} \sum_{i+j=m} d_{i}\left(E^{p q}\right)_{p t} d_{j}\left(E^{q r}\right)_{t r} \\
& =\sum_{i+j=m} d_{i}\left(E^{p q}\right)_{p q} d_{j}\left(E^{q r}\right)_{q r} \\
& =\sum_{i+j=m} f_{i}(p, q) f_{j}(q, r),
\end{aligned}
$$

i.e. $f \in T M_{s}(\rho, R)$.

Now we can prove the following

THEOREM 5.3. Let $P$ be a special subring of $M_{n}(R)$ with the relation $\rho$. Every $R$-derivation $d$ of order $s$ of $P$ has a unique representation:

(0) $d=\Delta(\underline{A}) * \Delta^{f}$,

where

(1) $\underline{A}=\left(A^{(m)}\right)_{m \in S \backslash 01}$ is a sequence of matrices $A^{(m)} \in P \cap M_{n}(Z(R))$ such that $A_{i i}^{(m)}=0$ for $i=1,2, \cdots, n$,

(2) $f$ is a transitive mapping of order $s$ from $\rho$ to $R$.

Proof. (I). Let $d \in D_{s}^{R}(P)$. We define matrices $A^{(1)}, A^{(2)}, \cdots$ inductively as follows :

and

$$
A_{p q}^{(1)}=d_{1}\left(E^{q q}\right)_{p q}
$$

where

$$
A_{p q}^{(m+1)}=d_{m+1}^{(m)}\left(E^{q q}\right)_{p q} \quad \text { for } \quad 1 \leqq m<s,
$$

$$
d^{(m)}=\left(\left[A^{(1)}, 1\right] * \cdots *\left[A^{(m)}, m\right]\right)^{-1} * d .
$$

Put $\delta=\left(\delta_{m}\right)_{m \in S}$, where $\delta_{0}=i d_{P}$ and $\delta_{m}=d_{m}^{(m)}$ for $m \geqq 1$. Let $\underline{A}=\left(A^{(m)}\right)_{m \in S \backslash 0\}}$ and let $f=\left(f_{m}\right)_{m \in S}$ be the sequence of mappings from $\rho$ to $R$ defined by

for all $m \in S, p \rho q$.

$$
f_{m}(p, q)=\delta_{m}\left(E^{p q}\right)_{p q}
$$

We show that $\underline{A}$ and $f$ satisfy conditions (0), (1) and (2) of this theorem. Observe first that 
a) $d_{k}^{(m)}=d_{k}^{(k)}$ for any $k \leqq m$,

b) $\delta$ is an $R$-derivation of order $s$ of $P$,

c) $d=\Delta(\underline{A}) * \delta$.

Now we prove that

d) $\delta_{m}\left(E^{q q}\right)_{p q}=0$ for $m \in S \backslash\{0\}$ and $p \neq q$.

In fact, for $m=1$ we have

$$
\begin{aligned}
\dot{\delta}_{1}\left(E^{q q}\right)_{p q} & =d_{1}^{(1)}\left(E^{q q}\right)_{p q} \\
& =\left(\left[A^{(1)}, 1\right]^{-1} * d\right)_{1}\left(E^{q q}\right)_{p q} \\
& =-\left[A^{(1)}, 1\right]_{1}\left(E^{q q}\right)_{p q}+d_{1}\left(E^{q q}\right)_{p q} \\
& =-\left(A^{(1)} E^{q q}-E^{q q} A^{(1)}\right)_{p q}+A_{p q}^{(1)} \\
& =-A_{p q}^{(1)}+A_{p q}^{(1)}=0
\end{aligned}
$$

and, if $m>1$ then

$$
\begin{aligned}
\delta_{m}\left(E^{q q}\right)_{p q} & =d_{m}^{(m)}\left(E^{q q}\right)_{p q} \\
& =\left(\left[A^{(m)}, m\right]^{-1} * d^{(m-1)}\right)_{m}\left(E^{q q}\right)_{p q} \\
& =\left(\sum_{i+j=m}\left[A^{(m)}, m\right]_{i}^{-1} \circ d_{j}^{(m-1)}\right)\left(E^{q q}\right)_{p q} \\
& =\left[A^{(m)}, m\right]_{m}^{-1}\left(E^{q q}\right)_{p q}+\left(\sum_{i=1}^{m-1} O d_{i}^{(m-1)}\right)\left(E^{q q}\right)_{p q}+d_{m}^{(m-1)}\left(E^{q q}\right)_{p q} \\
& =-\left(A^{(m)} E^{q q}-E^{q q} A^{(m)}\right)_{p q}+A_{p q}^{(m)} \\
& =-A_{p q}^{(m)}+A_{p q}^{(m)}=0 .
\end{aligned}
$$

Using b), d), a) and Lemma 5.1 we have

e) $\quad A_{p p}^{(m)}=d_{m}^{(m-1)}=d_{m}^{(m)}\left(E^{p p}\right)_{p p}=0$ for $m \geqq 2$.

Moreover, $A_{p p}^{(1)}=0$, since

$$
A_{p p}^{(1)}=d_{1}\left(E^{p p}\right)_{p p}=d_{1}\left(E^{p p} E^{p p}\right)_{p p}=A_{p p}^{(1)}+A_{p p}^{(1)} .
$$

Observe also that

f) $A^{(m)} \in M_{n}(Z(R)) \cap P$ (by Lemma 2.2),

and

g) $f$ is a transitive mapping of order $s$ from $\rho$ to $R$ (by b), d) and Lemma $5.2)$.

It remains to show that

h) $\delta=\Delta^{f}$.

If $X \in P, m \in S$ and $p \rho q$ then

$$
\delta_{m}(X)_{p q}=\delta_{m}\left(\sum_{i, j} \bar{X}_{i j} E^{i j}\right)_{p q}
$$


(II). Suppose that

$$
\begin{aligned}
& =\left(\sum_{i, j} \bar{X}_{i j} \delta_{m}\left(E^{i j}\right)\right)_{p q} \\
& =\sum_{i, j} X_{i j} \delta_{m}\left(E^{i j}\right)_{p q} \\
& =X_{p q} \delta_{m}\left(E^{p q}\right)_{p q} \quad(\text { by d) and Lemma } 1) \\
& =X_{p q} f_{m}(p, q) \\
& =\Delta_{m}^{f}(X)_{p q}, \quad \text { i.e }, \quad \delta=\Delta^{f} .
\end{aligned}
$$

$$
\Delta(\underline{A}) * \Delta^{f}=\Delta(\underline{B}) * \Delta^{g},
$$

where $\underline{A}, f$ and $\underline{B}, g$ satisfy conditions (1) and (2).

Then, for $p \neq q$,

$$
A_{p q}^{(1)}=\left(\Delta(\underline{A}) * \Delta^{f}\right)_{1}\left(E^{q q}\right)_{p q}=\left(\Delta(\underline{B}) * \Delta^{g}\right)_{1}\left(E^{q q}\right)_{p q}=B_{p q}^{(1)} .
$$

So $A^{(1)}=B^{(1)}$.

Suppose that $A^{(1)}=B^{(1)}, \cdots, A^{(m)}=B^{(m)}$ for some $m<s$. Then

hence

$$
\begin{aligned}
\Delta\left(0, \cdots 0, A^{(m+1)}, A^{(m+2)}, \cdots\right) * \Delta^{f} & =\left(\left[A^{(1)}, 1\right] * \cdots *\left[A^{(m)}, m\right]\right)^{-1} * \Delta(\underline{A}) * \Delta^{f} \\
& =\left(\left[B^{(1)}, 1\right] * \cdots *\left[B^{(m)}, m\right]\right)^{-1} * \Delta(\underline{B}) * \Delta^{g} \\
& =\Delta\left(0, \cdots, 0, B^{(m+1)}, B^{(m+2)}, \cdots\right) * \Delta^{g},
\end{aligned}
$$

and hence

$$
\begin{aligned}
A_{p q}^{(m+1)} & =\left(\Delta\left(0, \cdots, 0, A^{(m+1)}, A^{(m+2)}, \cdots\right) * \Delta^{f}\right)_{m+1}\left(E^{q q}\right)_{p q} \\
& =\left(\Delta\left(0, \cdots, 0, B^{(m+1)}, B^{(m+2)}, \cdots\right) * \Delta^{g}\right)_{m+1}\left(E^{q q}\right)_{p q} \\
& =B_{p q}^{(m+1)} \quad \text { for } \quad p \neq q
\end{aligned}
$$

$$
A^{(m+1)}=B^{(m+1)} \text {. }
$$

Therefore, by induction, $\underline{A}=\underline{B}$.

Further we have

$$
\begin{aligned}
\Delta^{f} & =\Delta(\underline{A})^{-1} *\left(\Delta(\underline{A}) * \Delta^{f}\right) \\
& =\Delta(\underline{B})^{-1} *\left(\Delta(\underline{B}) * \Delta^{g}\right)=\Delta^{g}
\end{aligned}
$$

hence, by Proposition 3.7, we obtain that $f=g$. This completes the proof.

\section{Corollaries to Theorem 5.3.}

Let $S^{\prime}$ be a segment of $N$ such that $S \subset S^{\prime}$ and let $s^{\prime}=\sup \left(S^{\prime}\right) \leqq \infty$. We say that a transitive mapping $f \in T M_{s}(\rho, R)$ is $s^{\prime}$-integrable if there exists a transitive mapping $f^{\prime} \in T M_{s}(\rho, R)$ such that $f_{m}^{\prime}=f_{m}$ for all $m \in S$.

As an immediate consequence of Theorem 5.3 we have 
COROLLARY 6.1. The following conditions are equivalent:

(1) Every R-derivation of order $s$ of $P$ is $s^{\prime}$-integrable,

(2) Every transitive mapping of order $s$ from $\rho$ to $R$ is $s^{\prime}$-integrable.

If $U$ is an ideal in $P$, then $U=\left[U_{i j}\right]$, where $U_{i j}$ are ideals of $R$ for any $i, j$ (see [7] Lemma 2.1). Therefore from Theorem 5.3 we get

CoRollary 6.2. If $d \in D_{s}^{R}(P)$ and $U$ is an ideal in $P$ then $d_{m}(U) \leqq U$ for all $m \in S$.

Observe also that from Theorem 5.3 follows

Corollary 6.3. If $d \in D_{s}^{R}(P)$ and $C$ is the center of $P$, then $d_{m}(C)=0$ for all $m \in S \backslash\{0\}$.

Denote by $I(P)$ the set of all matrices $A \in P$ such that $A_{p p}=0$ for all $p \in I_{n}$. It is easy to verify the following two lemmas.

LEMMA 6.4. The folowing conditions are equivalent:

(1) $I(P)$ is an ideal in $P$,

(2) $I(P)$ is a left-ideal in $P$,

(3) $I(P)$ is a right-ideal in $P$,

(4) $A B \in I(P)$ for all $A, B \in I(P)$,

(5) $A B-B A \in I(P)$ for all $A, B \in I(P)$,

(6) $A B-B A \in I(P)$ for all $A \in I(P), B \in P$,

(7) The relation $\rho$ is partial order.

LEMMA 6.5 The following two conditions are equivalent:

(1) $A B=0$ for all $A, B \in I(P)$,

(2) There do not exist three different elements $a, b, c \in I_{n}$ such that a $\rho b \rho c$.

Combining Lemma 6.4 with Theorem 5.3 and Lemma 3.2(1) we obtain

COROLLARY 6.6. Let $d \in D_{s}^{R}(P)$. If the relation $\rho$ is a partial order then $d_{m}(P) \subseteq I(P)$ for all $m \in S \backslash\{0\}$.

We end this section with

COROLLARY 6.7. Assume that there do not exist three different elements $a, b, c \in I_{n}$ such that a $\rho b \rho c$. Let $d=\left(d_{m}\right)_{m \in S}$ be a sequence of mappings from $P$ to 
$P$ such thot $d_{0}=i d_{P}$.

Then $d$ is an R-derivation of order $s$ of $P$ if and only if every mapping $d_{m}$ (for $m \in S \backslash\{0\}$ ) is an usual $R$-derivation of $P$.

Proof. If $d \in D_{s}^{R}(P)$ then, by Corollary 6.6 and Lemma 6.5, $d_{i}(A) d_{j}(B)=0$ for $i>0$ or $j>0$ and any $A, B \in P$. Therefore $d_{m}(A B)=A d_{m}(B)+d_{m}(A) B$, for any $m \in S \backslash\{0\}$ and $A, B \in P$. Conversely, if any $d_{m}$ is an usual $R$-derivation of $P$ then, by Corollary 6.6, $d_{m}(A) \cong I(P)$ for any $A \in P$, hence, by Lemma 6.5 , $d_{i}(A) d_{j}(B)=0$ for any $A, B \in P$ and $i>0$ or $j>0$. Therefore

$$
\begin{aligned}
d_{m}(A B) & =A d_{m}(B)+d_{m}(A) B \\
& =\sum_{i+j=m} d_{i}(A) d_{j}(B), \quad \text { i.e. } \quad d \in D_{s}^{R}(P) .
\end{aligned}
$$

\section{Integrable $R$-derivations.}

Let $S^{\prime}$ be a segment of $N$ such that $S \subset S^{\prime}$ and let $s^{\prime}=\sup \left(S^{\prime}\right) \leqq \infty$.

In the sequel we shall study $s^{\prime}$-integrable $R$-derivations of order $s$ of $P$.

In this section, we give some examples of such $R$-derivations and we show that in general there are non-integrable $R$-derivations.

Notice first that, by Corllary 6.1, we may reduce our investigations and to study only $s^{\prime}$-integrable transitive mappings of order $s$ from $\rho$ to $R$.

Observe also, that it suffices to consider the case where $\rho$ is a partial order. It follows from the following

LEMMA 7.1. The following conditions are equivalent:

(1) Every transitive mapping of order s from $\rho$ to $R$ is $s^{\prime}$-integrable,

(2) Every transitive mapping of order $s$ from $\rho^{\prime}$ to $R$ is $s^{\prime}$-integrable.

Proof. Denote by $W$ some fixed set of representatives of the cosets with respect to $\sim$.

$(1) \Rightarrow(2)$. Let $g \in T M_{s}\left(\rho^{\prime}, R\right)$. Consider the sequence $f=\left(f_{m}\right)_{m \in S}$ of mappings from $\rho$ to $Z(R)$ defined by $f_{m}(x, y)=g_{m}([x],[y])$ for all $m \in S$ and $x \rho y$. If $x \rho y \rho z$ then $[x] \rho^{\prime}[y] \rho^{\prime}[z]$ and we have

$$
\begin{aligned}
f_{m}(x, z) & =g_{m}([x],[z]) \\
& =\sum_{i+j=m} g_{i}([x],[y]) g_{j}([y],[z]) \\
& =\sum_{i+j=m} f_{i}(x, y) f_{j}(y, z)
\end{aligned}
$$

for all $m \subseteq S$. Therefore $f \in T M_{s}(\rho, R)$, and, by (1), there exists $f^{\prime} \in T M_{s^{\prime}}(\rho, R)$ 
such that $f_{m}^{\prime}=f_{m}$ for all $m \in S$.

$$
\text { Put } g_{i}^{\prime}([a],[b])=f_{i}^{\prime}(a, b) \quad \text { for } \quad i \in S^{\prime} \quad \text { and } a, b \in W .
$$

Then $g^{\prime}=\left(g_{i}^{\prime}\right)_{i \in S^{\prime}}$ is a transitive mapping of order $s^{\prime}$ from $\rho^{\prime}$ to $R$. Indeed, if $[a] \rho^{\prime}[b] \rho^{\prime}[c]$, then $a \rho b \rho c$ and we have

$$
\begin{aligned}
g_{i}^{\prime}([a],[c]) & =f_{i}^{\prime}(a, c) \\
& =\sum_{p+q=i} f_{p}^{\prime}(a, b) f_{q}^{\prime}(b, c) \\
& =\sum_{p+q=i} g_{p}^{\prime}([a],[b]) g_{q}^{\prime}([b],[c]) \quad \text { for all } i \in S^{\prime} .
\end{aligned}
$$

Moreover, if $m \in S,[a] \rho^{\prime}[b]$ then

$$
g_{m}^{\prime}([a],[b])=f_{m}^{\prime}(a, b)=f_{m}(a, b)=g_{m}([a],[b]),
$$

i.e. $g_{m}^{\prime}=g_{m}$ for all $m \in S$.

$(2) \Rightarrow(1)$. Let $f \in T M_{s}(\rho, R)$. We define the element $g \in T M_{s}\left(\rho^{\prime}, R\right)$ by

$$
g_{m}([a],[b])=f_{m}(a, b),
$$

where $m \in S$ and $a, b \in W$.

Let $g^{\prime}$ be such an element in $T M_{s^{\prime}}\left(\rho^{\prime}, R\right)$ that $g_{m}^{\prime}=g_{m}$ for all $m \in S$. We shall construct (by induction) a sequence $f^{\prime} \in T M_{s^{\prime}}(\rho, R)$ such that

$$
f_{m}^{\prime}=f_{m} \quad \text { for all } m \in S,
$$

and

$$
f_{k}^{\prime}(a, b)=g_{k}^{\prime}([a],[b]) \quad \text { for all } a, b \in W \text { and } k \in S^{\prime} .
$$

If $t \leqq s$ then we put $f_{t}^{\prime}=f_{t}$.

Now let $s \leqq t<s^{\prime}$ and assuume that $\left(f_{0}^{\prime}, f_{1}^{\prime}, \cdots, f_{t}^{\prime}\right) \in T M_{t}(\rho, R)$ and the mappings $f_{0}^{\prime}, f_{1}^{\prime}, \cdots, f_{t}^{\prime}$ satisfy the condition (ii). If $x \rho y$ then we put

$$
\begin{aligned}
f_{t+1}^{\prime}(x, y)= & g_{t+1}^{\prime}([a],[b]) \\
= & \sum_{i=1}^{t} f_{i}^{\prime}(x, a) f_{t+1-i}^{\prime}(a, y) \\
& -\sum_{i=1}^{t} f_{i}^{\prime}(y, b) f_{t+1-i}^{\prime}(b, y) \\
& +\sum_{i=1}^{t} f_{i}^{\prime}(a, b) f_{t+1-i}^{\prime}(b, y),
\end{aligned}
$$

where $a, b$ are elements of $W$ such that $x \sim a, y \sim b$. Lemma 3.2 implies that $f_{t+1}^{\prime}(a, b)=g_{t+1}^{\prime}([a],[b])$ for $a, b \in W$.

It remains to show that 


$$
f_{t+1}^{\prime}(x, z)-f_{t+1}^{\prime}(x, y)-f_{t+1}^{\prime}(y, z)=\sum_{i=1}^{t} f_{i}^{\prime}(x, y) f_{t+1-i}^{\prime}(y, z)
$$

for $x \rho y \rho z$.

For this purpose we introduce the following notices:

$$
\begin{aligned}
& \left(x_{1}, x_{2}, x_{3}\right)=\sum_{i=1}^{t} f_{i}^{\prime}\left(x_{1}, x_{2}\right) f_{t+1-i}^{\prime}\left(x_{2}, x_{3}\right) \quad \text { for } x_{1} \rho x_{2} \rho x_{3}, \\
& \begin{aligned}
A\left(x_{1}, x_{2}, x_{3}, x_{4}\right)= & \left(x_{2}, x_{3}, x_{4}\right)-\left(x_{1}, x_{3}, x_{4}\right) \\
& +\left(x_{1}, x_{2}, x_{4}\right)-\left(x_{1}, x_{2}, x_{3}\right) \text { for } x_{1} \rho x_{2} \rho x_{3} \rho x_{4} .
\end{aligned}
\end{aligned}
$$

Observe that

(iii)

$$
A\left(x_{1}, x_{2}, x_{3}, x_{4}\right)=0
$$

In fact,

$$
\begin{aligned}
A\left(x_{1}, x_{2}, x_{3}, x_{4}\right)= & -\sum_{i=1}^{t}\left(f_{i}^{\prime}\left(x_{1}, x_{3}\right)-f_{i}^{\prime}\left(x_{2}, x_{3}\right)\right) f_{t+1-i}^{\prime}\left(x_{3}, x_{4}\right) \\
& +\sum_{i=1}^{t} f_{i}^{\prime}\left(x_{1}, x_{2}\right)\left(f_{t+1-i}^{\prime}\left(x_{2}, x_{4}\right)-f_{t+1-i}^{\prime}\left(x_{2}, x_{3}\right)\right) \\
= & -\sum_{i=1}^{t} f_{i}^{\prime}\left(x_{1}, x_{2}\right) f_{t+1-i}^{\prime}\left(x_{3}, x_{4}\right) \\
& -\sum f_{p}^{\prime}\left(x_{1}, x_{2}\right) f_{q}^{\prime}\left(x_{2}, x_{3}\right) f_{r}^{\prime}\left(x_{3}, x_{4}\right) \\
& +\sum_{i=1}^{t} f_{i}^{\prime}\left(x_{1}, x_{2}\right) f_{t+1-i}^{\prime}\left(x_{3}, x_{4}\right) \\
& +\sum f_{p}^{\prime}\left(x_{1}, x_{2}\right) f_{q}^{\prime}\left(x_{2}, x_{3}\right) f_{r}^{\prime}\left(x_{3}, x_{4}\right) \\
= & 0 .
\end{aligned}
$$

Observe also that if $a, b, c$ are such elements of $W$ that $a \rho b \rho c$ then, by (ii), we have

$$
g_{t+1}^{\prime}([a],[c])-g_{t+1}^{\prime}([a],[b])-g_{t+1}^{\prime}([b],[c])=(a, b, c) .
$$

In fact, since $g^{\prime} \in T M_{s^{\prime}}\left(\rho^{\prime}, R\right)$ we have

$$
\begin{aligned}
g_{t+1}^{\prime}( & {[a],[c])-g_{t+1}^{\prime}([a],[b])-g_{t+1}^{\prime}([b],[c]) } \\
& =\sum_{i=1}^{t} g_{i}^{\prime}([a],[b]) g_{t+1-t}^{\prime}([b],[c]) \\
& =\sum_{i=1}^{t} f_{i}^{\prime}(a, b) f_{t+1-i}^{\prime}(b, c) \\
& =(a, b, c) .
\end{aligned}
$$

Now, let $x \rho y \rho z$ and let $a, b, c$ be such elements of $W$ that $a \sim x, b \sim y, c \sim z$. Then, by (iii), (iv) and by the fact thet $(y, y, z)=0$ (Lemma 3.2) we obtain 


$$
\begin{aligned}
f_{t+1}^{\prime}(x, z) & -f_{t+1}^{\prime}(x, y)-f_{t+1}^{\prime}(y, z) \\
= & (a, b, c) \\
& +(x, a, z)-(z, c, z)+(a, c, z) \\
& -(x, a, y)+(y, b, y)-(a, b, y) \\
& -(y, b, z)+(z, c, z)-(b, c, z) \\
= & ((a, y, z)-(x, y, z)+(x, a, z)-(x, a, y)) \\
& -((b, c, z)-(a, c, z)+(a, b, z)-(a, b, c)) \\
& +((b, y, z)-(a, y, z)+(a, b, z)-(a, b, y)) \\
& -((b, y, z)-(y, y, z)+(y, b, z)-(y, b, y)) \\
& +(x, y, z)-(y, y, z) \\
= & A(x, a, y, z)-A(a, b, c, z)+A(a, b, y, z)-A(y, b, y, z) \\
& +(x, y, z)-(y, y, z) \\
= & (x, y, z)-(y, y, z) \\
= & (x, y, z) .
\end{aligned}
$$

This completes the proof.

EXAMPLE 7.2. Let $P$ be such as in Example 4.3. Since $D_{s}^{R}(P)=I D_{s}^{R}(P)$ then every $R$-derivation of order $s$ of $P$ is $s^{\prime}$-integrable (for any $s^{\prime}$ ).

ExAMPLE 7.3. Let $P=M_{4}(R)$, where

$$
\rho=\downarrow_{4}^{1} \begin{aligned}
& 1 \\
& 4
\end{aligned} \quad \text { i.e. } P=\left[\begin{array}{cccc}
R & 0 & R & R \\
0 & R & R & R \\
0 & 0 & R & 0 \\
0 & 0 & 0 & R
\end{array}\right] .
$$

There exist $R$-derivations of order $s$ of $P$ which are not inner $([7])$. But, by Corollary 6.1 and Example 3.5, every $R$-derivation of order $s$ of $P$ is $s^{\prime}$-integrable, for any $s^{\prime} \leqq \infty$ (see also Corollary 6.7).

EXAMPLE 7.4. Consider the following relation $\rho$ on the set $I_{17}$ 


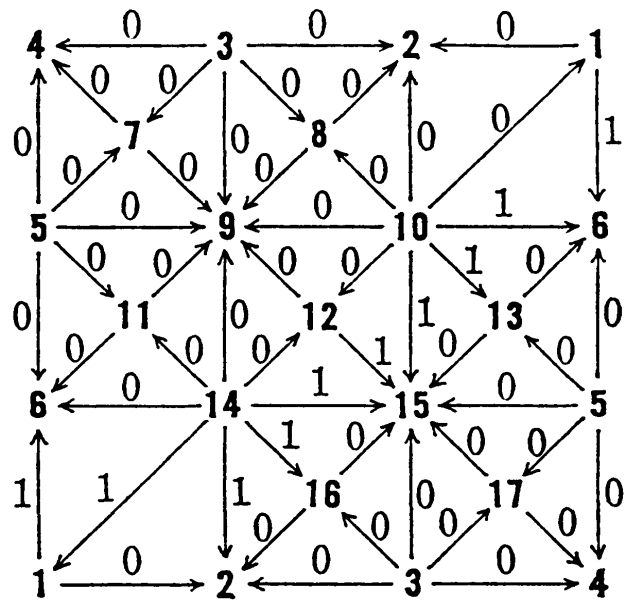

(see [7] Section 5).

Let $R=Z_{2}$ and let $f_{1}: \rho \rightarrow Z_{2}$ be the usual transitive mapping from $\rho$ to $Z_{2}$ defined by the numbers at the arrows (for example $f_{1}(14,1)=1, f_{1}(10,2)=0$ ).

Let $f_{0}(a, b)=1$ for all $a \rho b$. Then $f=\left(f_{0}, f_{1}\right)$ is a transitive mapping of order 1 from $\rho$ to $Z_{2}$. We show that $f$ is not 2 -integrable. Suppose that there exists $f_{2}: \rho \rightarrow Z_{2}$ such that

$$
f_{2}(a, c)=f_{2}(a, b)+f_{2}(b, c)+f_{1}(a, b) f_{1}(b, c),
$$

for any $a \rho b \rho c$.

Denote $f_{2}(a, b)$ by $(a, b)$. Then we have

$$
\begin{aligned}
1= & f_{1}(14,1) f_{1}(1,6) \\
= & (14,6)+(14,1)+(1,6) \\
= & {[(14,12)+(10,12)+(10,1)+(1,2)+(3,2)+(3,4)+(5,4)+(5,6)] } \\
& \left.+[(1,2)+(3,2)+(3,4)+(5,4)+(5,6)+(1,6)+(10,1)+(10,12)+(14,12)]+\cdot^{\prime} 1,6\right) \\
= & 0 ?
\end{aligned}
$$

The above example and Corollary 6.1 show that there exist non-integiable $R$ derivations of $P$.

\section{A necessary condition for $\mathbf{s}^{\prime}$-integrability.}

Let $\Gamma=\Gamma(\rho)=\left(I_{n}^{\prime}, \rho^{\prime}\right)$ be the graph of the relation $\rho$ (see Section 2), and $f \in T M_{s}\left(\rho^{\prime}, R\right)$.

If $a, b, c$ are such elements in $I_{n}^{\prime}$ that $a \rho^{\prime} b \rho^{\prime} c$ then by $t(a, b, c)$ we denote the element $(a, c)-(a, b)-(b, c)$ of $C_{1}(\Gamma)$, and by $\bar{f}_{m+1}(a, b, c)$, for $m \in S$, we denote the element

$$
\sum_{i=1}^{m} f_{i}(a, b) f_{m+1-i}(b, c)
$$


of $Z(R)$.

For example :

$$
\begin{aligned}
& \bar{f}_{1}(a, b, c)=0, \\
& \bar{f}_{2}(a, b, c)=f_{1}(a, b) f_{1}(b, c), \\
& \bar{f}_{3}(a, b, c)=f_{1}(a, b) f_{2}(b, c)+f_{2}(a, b) f_{1}(b, c) .
\end{aligned}
$$

Consider the following equality (in the group $C_{1}(\Gamma)$ ):

$$
\sum_{i=1}^{k} z_{i} t\left(a_{i}, b_{i}, c_{i}\right)=0,
$$

where $k \in N, z_{1}, \cdots, z_{k} \in Z$ and $a_{i} \rho^{\prime} b_{i} \rho^{\prime} c_{i}$ for $i=1,2, \cdots, k$.

Definition 8.1. Let $s<\infty$. We say that $\Gamma$ is an s-graph over $R$ if for any transitive mapping $f$ of order $s$ from $\rho^{\prime}$ to $R$ and for any equality of the form (*) holds

$$
\sum_{i=1}^{k} z_{i} \bar{f}_{s+1}\left(a_{i}, b_{i}, c_{i}\right)=0
$$

For example, $\Gamma$ is a 1 -graph over $R$ if for every usual transitive mapping $\varphi: \rho^{\prime} \rightarrow Z(R)$ and for every equality (*) holds

$$
\sum_{i=1}^{k} z_{i} \varphi\left(a_{i}, b_{i}\right) \varphi\left(b_{i}, c_{i}\right)=0,
$$

and $\Gamma$ is a 2-graph over $R$ if for every $f=\left(f_{0}, f_{1}, f_{2}\right) \in T M_{2}\left(\rho^{\prime}, R\right)$ and for every equality (*) holds

$$
\sum_{i=0}^{k} z_{i}\left(f_{1}\left(a_{i}, b_{i}\right) f_{2}\left(b_{i}, c_{i}\right)+f_{2}\left(a_{i}, b_{i}\right) f_{1}\left(b_{i}, c_{i}\right)\right)=0 .
$$

In Section 9 we prove that every graph $\Gamma$ is a 1 -graph and is a 2 -graph over an arbitrary ring $R$.

EXAMPLE 8.2. Let

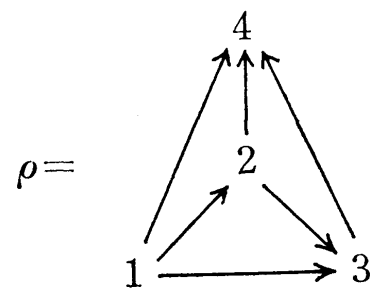

We show that $\Gamma=\left(I_{4}, \rho\right)$ is an $s$-graph over an arbitrary ring $R$, for any $s \in N$.

Observe, that for $\Gamma$ we have only one equality of the form $(*)$. Namely,

$$
[(1,4)-(1,2)-(2,4)]-[(1,3)-(1,2)-(2,3)]
$$




$$
+[(2,4)-(2,3)-(3,4)]-[(1,4)-(1,3)-(3,4)]=0,
$$

i.e. $t(1,2,4)-t(1,2,3)+t(2,3,4)-t(1,3,4)=0$.

If $s \in N, f \in T M_{s}(\rho, R)$, then we have

$$
\begin{aligned}
\bar{f}_{s+1}(1,2,4)-\bar{f}_{s+1}(1,2,3)+\bar{f}_{s+1}(2,3,4)-\bar{f}_{s+1}(1,3,4) \\
=\sum_{k=1}^{s}\left[f_{k}(1,2) f_{s+1-k}(2,4)-f_{k}(1,2) f_{s+1-k}(2,3)\right. \\
\left.\quad+f_{k}(2,3) f_{s+1-k}(3,4)-f_{k}(1,3) f_{s+1-k}(3,4)\right] \\
=\sum_{k=1}^{s} f_{k}(1,2)\left(f_{s+1-k}(3,4)+\sum_{\substack{p+q=s-k+1 \\
p \geq 1, q \geq 1}} f_{p}(3,4) f_{q}(2,3)\right) \\
\quad-\sum_{k=1}^{s}\left(f_{k}(1,2)+\sum_{\substack{p+q=k \\
p \geq 1, q \geq 1}} f_{p}(1,2) f_{q}(2,3)\right) f_{s+1-k}(3,4)=0 .
\end{aligned}
$$

Now we prove a necessary condition for any $R$-derivation of order $s$ of $P$ to be $(s+1)$-integrable.

Proposition 8.3. Let $P=M_{n}(R)_{\rho}$. If every $R$-derivation of order $s$ of $P$ is $(s+1)$-integrable then $\Gamma=\Gamma(\rho)$ is an s-graph.

Proof. Consider in $C_{1}(\Gamma)$ the equality of the form (*) and let $f \in T M_{s}\left(\rho^{\prime}, R\right)$. There exists, by Corollary 6.1 and Lemma 7.1, a transitive mapping $f^{\prime} \in T M_{s+1}\left(\rho^{\prime}\right.$, $R$ ) such that $f_{m}^{\prime}=f_{m}$ for all $m=0,1, \cdots, s$. Observe that, for $i=1,2, \cdots, k$, we have

$$
f_{s+1}^{\prime}\left(a_{i}, c_{i}\right)-f_{s+1}^{\prime}\left(a_{i}, b_{i}\right)-f_{s+1}^{\prime}\left(b_{i}, c_{i}\right)=\bar{f}_{s+1}\left(a_{i}, b_{i}, c_{i}\right)
$$

Let $\varphi: C_{1}(\Gamma) \rightarrow Z(R)$ be the group homomorphism defined (for free generators) by $\varphi(a, b)=f_{s+1}^{\prime}(a, b)$.

Then we have

$$
\begin{aligned}
\sum_{i=1}^{k} z_{i} \bar{f}_{s+1}\left(a_{i}, b_{i}, c_{i}\right) & =\sum_{i=1}^{k} z_{i}\left(f_{s+1}^{\prime}\left(a_{i}, c_{i}\right)-f_{s+1}^{\prime}\left(a_{i}, b_{i}\right)-f_{s+1}^{\prime}\left(b_{i}, c_{i}\right)\right) \\
& =\sum_{i=1}^{k} z_{i}\left(\varphi\left(a_{i}, b_{i}\right)-\varphi\left(a_{i}, b_{i}\right)-\varphi\left(b_{i}, c_{i}\right)\right) \\
& =\varphi\left(\sum_{i=1}^{k} z_{i} t\left(a_{i}, b_{i}, c_{i}\right)\right) \\
& =\varphi(0) \\
& =0 . \quad \text { This completes the proof. }
\end{aligned}
$$

We obtain some examples of $s$-graphs by the following 
LEMMA 8.4. If $H_{2}(\Gamma)=0$ then $\Gamma$ is an s-graph over $R$ for any natural s.

Proof. Suppose that in $C_{1}(\Gamma)$ the equality $(*)$ holds, and let $f \in T M_{s}\left(\rho^{\prime}, R\right)$. We must to show that $\sum_{i=1}^{k} z_{i} \bar{f}_{s+1}\left(a_{i}, b_{i}, c_{i}\right)=0$.

Consider the group homomorphism $\varphi: C_{2}(\Gamma) \rightarrow R$ defined for free-generators by $\varphi(a, b, c)=,\bar{f}_{s+1}(a, b, c)$. Since $\sum_{i=1}^{k} z_{i}\left(a_{i}, b_{i}, c_{i}\right) \in \operatorname{Ker} \partial_{2}$ and Ker $\partial_{2}=\operatorname{Im} \partial_{3}$ (see Section 2) then

$$
\sum_{i=1}^{k} z_{i}\left(a_{i}, b_{i}, c_{i}\right)=\sum_{j=1}^{l} u_{j}\left[\left(x_{j}, y_{j}, w_{j}\right)-\left(x_{j}, y_{j}, t_{j}\right)+\left(x_{j}, w_{j}, t_{j}\right)-\left(y_{j}, w_{j}, t_{j}\right)\right]
$$

for some $u_{1}, \cdots, u_{l} \in Z$ and $x_{j} \rho^{\prime} y_{j} \rho^{\prime} w_{j} \rho^{\prime} t_{j}, j=1,2, \cdots, l$.

Therefore, by Example 8.2, we have

$$
\begin{aligned}
\sum_{i=1}^{k} z_{i} \bar{f}_{s+1}\left(a_{i}, b_{i}, c_{i}\right)= & \varphi\left(\sum_{i=1}^{k} z_{i}\left(a_{i}, b_{i}, c_{i}\right)\right) \\
= & \sum_{j=1}^{l} u_{j}\left[\bar{f}_{s+1}\left(x_{j}, y_{j}, w_{j}\right)-\bar{f}_{s+1}\left(x_{j}, y_{j}, t_{j}\right)\right. \\
& \left.+\bar{f}_{s+1}\left(x_{j}, w_{j}, t_{j}\right)-\bar{f}_{s+1}\left(y_{j}, w_{j}, t_{j}\right)\right] \\
= & \sum_{j=1}^{l} u_{j} 0=0 . \quad \text { This completes the proof. }
\end{aligned}
$$

REMARK 8.5. The necessary condition for any $R$-derivation of order $s$ of $P$ to be $(s+1)$-integrable given in Proposition 8.3 is not sufficient. For example. let $\Gamma$ be such as in Example 7.4. Then $\Gamma$ is one-dimensional triangulation of the projective plane, and therefore $H_{2}(\Gamma)=0$ (see [3]]. So, by Lemma 8.4, $\Gamma$ is a 1-graph over $Z_{2}$. But, by Example 7.4, there exists an $R$-derivation $d$ of order 1 of $P=M_{n}(R)_{\rho}$ (where $R=Z_{2}$ ) such that $d$ is not 2-integrable.

THEOREM 8.6. Let $P$ be a special subring of $M_{n}(R)$ with the relation $\rho$, and let $\Gamma=\Gamma(\rho)$ and $s<s^{\prime} \leqq \infty$. If $H_{2}(\Gamma)=0$ and $H_{1}(\Gamma)$ is a free abelian group then every $R$-derivation of order $s$ of $P$ is $s^{\prime}$-integrable.

Proof. It follows from Corollary 6.1 and Lemma 7.1 that it is sufficient to prove that every transitive mapping of order $s$ from $\rho^{\prime}$ to $R$ is $(s+1)$-integrable.

Let $f \in T M_{s}\left(\rho^{\prime}, R\right)$ and consider a group homomorphism $\varphi: \operatorname{Im} \partial_{2} \rightarrow Z(R)$ defined (for generators) by $\varphi\left(\partial_{2}(a, b, c)\right)=-\bar{f}_{s+1}(a, b, c)$. Observe that, by Lemma 8.4, $\varphi$ is a well defined mapping. Since $H_{1}(\Gamma)$ is free then $\varphi$ we can extend to a group homomorphism $\varphi^{\prime}: \operatorname{Ker} \partial_{1} \rightarrow Z(R)$. Further, by [7] Lemma 5.5, we can extend $\varphi^{\prime}$ to a group homomorphism $\varphi^{\prime \prime}: C_{1}(\Gamma) \rightarrow Z(R)$. Put $f_{s+1}(a, b)=\varphi^{\prime \prime}(a, b)$ for all $a \rho^{\prime} b$. We show that, for any $a \rho^{\prime} b \rho^{\prime} c$, holds 


$$
\begin{aligned}
f_{s+1}(a, c) & =\sum_{i+j=s+1} f_{i}(a, b) f_{j}(b, c) \\
& =f_{s+1}(a, b)+f_{s+1}(b, c)+\sum_{i=1}^{s} f_{i}(a, b) f_{s+1-i}(b, c) .
\end{aligned}
$$

In fact

$$
\begin{aligned}
f_{s+1}( & (a, b)-f_{s+1}(a, b)-f_{s+1}(b, c) \\
& =\varphi^{\prime \prime}(a, c)-\varphi^{\prime \prime}(a, b)-\varphi^{\prime \prime}\left(b, c^{\prime} ;\right. \\
& =-\varphi^{\prime \prime}\left(\partial_{2}(a, b, c)\right) \\
& =-\varphi\left(\partial_{2}(a, b, c)\right) \\
& =\bar{f}_{s+1}(a, b, c) \\
& =\sum_{i=1}^{s} f_{i}(a, b) f_{s+1-i}(b, c) .
\end{aligned}
$$

Therefore $\left(1, f_{1}, \cdots, f_{s}, f_{s+1}\right)$ is a transitive mapping of order $(s+1)$ from $\rho^{\prime}$ to $R$, i.e. $f$ is $(s+1)$-integrable. This completes the proof.

\section{9. s-graphs.}

In this section, using some additional properties of $s$-graphs, we describe (for fixed $s<s^{\prime}$ ) a new class of special subrings of $M_{n}(R)$ in which every $R$ derivation of order $s$ is $s^{\prime}$-integrable.

Let $\Gamma=\left(I_{n}^{\prime}, \rho^{\prime}\right)$ be the graph of the relation $\rho$ and let $W(\Gamma)=Z\left[X_{(a, b)} ; a \rho^{\prime} b\right]$ be the ring of polynomials over $Z$ in commuting indeterminates, one for each pair $(a, b)$, where $a \rho^{\prime} b$. Denote by $T(\Gamma)$ the ring $W(\Gamma) / I(\Gamma)$, where $I(\Gamma)$ is the ideal in $W(\Gamma)$ generated by all elements of the form

$$
X_{(a, c)}-X_{(a, b)}-X_{(b, c)}
$$

for $a \rho^{\prime} b \rho^{\prime} c$.

Moreover, denote by $\langle a, b\rangle$ the coset of the element $X_{(a, b)}$ in $T(\Gamma)$.

The following lemma plays a basic role in our further considerations.

LEMMA 9.1. Let $n$ be a power of a prime number $p$. If in the proup $C_{1}(\Gamma)$ holds the equality of the form (*), then in the ring $T(\Gamma)$ the following equality holds

$$
\sum_{i=1}^{k} z_{i} \sum_{j=1}^{n-1}(1 / p)\left(\begin{array}{c}
n \\
j
\end{array}\right)\left\langle a_{i}, b_{i}\right\rangle^{j}\left\langle b_{i}, c_{i}\right\rangle^{n-j}=0
$$

Proof. Observe that the equality $(*)$ is equivalent to an equality of the form

$$
\sum_{i=1}^{u}\left(a_{i}^{\prime}, c_{i}^{\prime}\right)+\sum_{j=1}^{v}\left(\left(a_{j}^{\prime \prime}, b_{j}^{\prime \prime}\right)+\left(b_{j}^{\prime \prime}, c_{j}^{\prime \prime}\right)\right)
$$




$$
=\sum_{j=1}^{v}\left(a_{j}^{\prime \prime}, c_{j}^{\prime \prime}\right)+\sum_{i=1}^{u}\left(\left(a_{i}^{\prime}, b_{i}^{\prime}\right)+\left(b_{i}^{\prime}, c_{i}^{\prime}\right)\right),
$$

where $a_{i}^{\prime} \rho^{\prime} b_{i}^{\prime} \rho^{\prime} c_{i}^{\prime}, a_{j}^{\prime \prime} \rho^{\prime} b_{j}^{\prime \prime} \rho^{\prime} c_{j}^{\prime \prime}$ for some integers $u, v$ and $i=1, \cdots, u, j=1, \cdots, v$.

Hence it suffices to prove that, in the ring $T(\Gamma)$, we have

$$
\begin{aligned}
\sum_{i=1}^{u} \sum_{k=1}^{n-1}(1 / p)\left(\begin{array}{l}
n \\
k
\end{array}\right)\left\langle a_{i}^{\prime}, b_{i}^{\prime}\right\rangle^{k}\left\langle b_{i}^{\prime}, c_{i}^{\prime}\right\rangle^{n-k} & \\
= & \sum_{j=1}^{v} \sum_{k=1}^{n-1}(1 / p)\left(\begin{array}{l}
n \\
k
\end{array}\right)\left\langle a_{j}^{\prime \prime}, b_{j}^{\prime \prime}\right\rangle^{k}\left\langle b_{j}^{\prime \prime}, c_{j}^{\prime \prime}\right\rangle^{n-k} .
\end{aligned}
$$

Let $\alpha, \beta: C_{1}(\Gamma) \rightarrow W(\Gamma)$ be the group homomorphisms defined, for free generators, as follows :

and

$$
\alpha(a, b)=X_{(a, b)}
$$

$$
\beta(a, b)=X_{(a, b)}^{n} .
$$

Further we denote $X_{(a, b)}$ by $(a, b)$ (for all $a \rho^{\prime} b$ ).

Applying $\alpha$ to the equality (**) we obtain the equality $(* *)$ in the ring $W(\Gamma)$. Applying $\beta$ to the equality (**) we obtain the following equality in $W(\Gamma)$ :

$$
\begin{aligned}
& \sum_{i=1}^{u}\left(a_{i}^{\prime}, c_{i}^{\prime}\right)^{n}+\sum_{j=1}^{v}\left(\left(a_{j}^{\prime \prime}, b_{j}^{\prime \prime}\right)^{n}+\left(b_{j}^{\prime \prime}, c_{j}^{\prime \prime}\right)^{n}\right) \\
& =\sum_{j=1}^{v}\left(a_{j}^{\prime \prime}, c_{j}^{\prime \prime}\right)^{n}+\sum_{i=1}^{u}\left(\left(a_{i}^{\prime}, b_{i}^{\prime}\right)^{n}+\left(b_{i}^{\prime}, c_{i}^{\prime}\right)^{n}\right) .
\end{aligned}
$$

Let

and

$$
\begin{aligned}
& A_{i}=\left(a_{i}^{\prime}, c_{i}^{\prime}\right), \\
& B_{i}=\left(a_{i}^{\prime}, b_{i}^{\prime}\right)+\left(b_{i}^{\prime}, c_{i}^{\prime}\right) \text { for } \quad i=1,2, \cdots, u,
\end{aligned}
$$

$$
\begin{aligned}
& C_{j}=\left(a_{j}^{\prime \prime}, c_{j}^{\prime \prime}\right), \\
& D_{j}=\left(a_{j}^{\prime \prime}, b_{j}^{\prime \prime}\right)+\left(b_{j}^{\prime \prime}, c_{j}^{\prime \prime}\right) \text { for } j=1,2, \cdots, v .
\end{aligned}
$$

Rise both sides of the equality $(* *)$ in $W(\Gamma)$ to the $n$-th power and apply (1). Then we have

$$
\begin{aligned}
& \sum_{i=1}^{n} \sum_{k=1}^{n-1}\left(\begin{array}{l}
n \\
k
\end{array}\right)\left(a_{i}^{\prime}, b_{i}^{\prime}\right)^{k}\left(b_{i}^{\prime}, c_{i}^{\prime}\right)^{n-k}-\sum_{j=1}^{v} \sum_{k=1}^{n-1}\left(\begin{array}{l}
n \\
k
\end{array}\right)\left(a_{j}^{\prime \prime}, b_{j}^{\prime \prime}\right)^{k}\left(b_{j}^{\prime \prime}, c_{j}^{\prime \prime}\right)^{n-k} \\
& =\sum_{\substack{i_{1}+\cdots+i_{u}=n \\
i_{1}, \cdots, i_{u} \neq n}}\left(i_{1}, \cdots, i_{u}\right)\left\{A_{1}^{i_{1}} \cdots A_{u}^{i u}-B_{1}^{i_{1}} \cdots B_{u}^{i u}\right] \\
& +\sum_{\substack{j_{1}+\cdots+j_{v=n} \\
j_{1}, \cdots, j v \neq n}}\left(j_{1}, \cdots, j_{v}\right)\left[D_{1}^{j_{1}} \cdots D_{v}^{j_{v}}-C_{1}^{j_{1}} \cdots C_{v}^{j_{v}}\right] \\
& +\sum_{k=1}^{n-1}\left(\begin{array}{l}
u \\
k
\end{array}\right)\left[\left(\sum_{i=1}^{u} A_{i}\right)^{k}\left(\sum_{j=1}^{v} D_{j}\right)^{n-k}-\left(\sum_{i=1}^{u} B_{i}\right)^{k}\left(\sum_{j=1}^{v} C_{j}\right)^{n-k}\right],
\end{aligned}
$$


where $\left(i_{1}, \cdots, i_{u}\right),\left(i_{1}, \cdots j_{v}\right)$ are Newton symbols, i.e.

$$
\left(n_{1}, \cdots, n_{k}\right)=\frac{\left(n_{1}+\cdots+n_{k}\right) !}{n_{1} ! \cdots n_{k} !} \quad \text { for integers } n_{1}, \cdots, n_{k} \geqq 0 \text {. }
$$

Since $n$ is a power of a prime number $p$ then every Newton symbol in the equality (2) is divisible by $p$, and therefore, since $W(\Gamma)$ is a ring with no $Z$ torsion, we can divide both sides of the equality (2) by $p$. We obtain the new equality in $W(\Gamma)$, we denote it by (3).

Observe, that the right side of the equality (3) is an element of the ideal $I(\Gamma)$. Therefore, in the ring $T(\Gamma)$, we have the equality $(* * *)$. This completes the proof.

As a consequence of Lemma 9.1 we obtain

THEOREM 9.2. Every graph $\Gamma$ is a 1-graph over an arbitrary ring $R$.

Observe, that this theorem is obvious if $R$ is a 2-torsion-free ring. In fact. Let $f_{1}: \rho^{\prime} \rightarrow Z(R)$ be an usual transitive mapping and suppose that in $C_{1}(\Gamma)$ the equality of the form $(*)$ holds. Consider the group homomorphism $\varphi: C_{1}(\Gamma) \rightarrow$ $Z(R)$ such that $\varphi(a, b)=f_{1}(a, b)^{2}$, for all $a \rho^{\prime} b$. Then we have

$$
\begin{aligned}
& 2 \sum_{i=1}^{k} z_{i} f_{1}\left(a_{i}, b_{i}\right) f_{1}\left(b_{i}, c_{i}\right) \\
& \quad=\sum_{i=1}^{k} z_{i}\left[\left(f_{1}\left(a_{i}, b_{i}\right)+f_{1}\left(b_{i}, c_{i}\right)\right)^{2}-f_{1}^{2}\left(a_{i}, b_{i}\right)-f_{1}^{2}\left(b_{i}, c_{i}\right)\right] \\
& \quad=\sum_{i=1}^{k} z_{i}\left[\varphi\left(a_{i}, c_{i}\right)-\varphi\left(a_{i}, b_{i}\right)-\varphi\left(b_{i}, c_{i}\right)\right] \\
& \quad=\varphi\left(\sum_{i=1}^{k} z_{i} t\left(a_{i}, b_{i}, c_{i}\right)\right) \\
& =\varphi(0) \\
& =0 .
\end{aligned}
$$

Proof of Theorem 9.2. Let $f \in T M_{1}\left(\rho^{\prime}, R\right)$ and suppose that in $C_{1}(\Gamma)$ the equality of the form $(*)$ holds. Let $h: W(\Gamma) \rightarrow Z(R)$ be the ring homomorphism such that $h\left(X_{(a, b)}\right)=f_{1}(a, b)$ for all $a \rho^{\prime} b$. Since $f_{1}$ is an usual transitive mapping then $h$ induces a ring homomorphism $\bar{h}: T(\Gamma) \rightarrow Z(R)$ such that $\bar{h}(\langle a, b\rangle)=f_{1}(a, b)$. From Lemma 9.1, for $n=2$, we have

$$
\begin{aligned}
\sum_{i=1}^{k} z_{i} f_{1}\left(a_{i}, b_{i}\right) f_{1}\left(b_{i}, c_{i}\right) & =\bar{h}\left(\sum_{i=1}^{k} z_{i}\left\langle a_{i}, b_{i}\right\rangle\left\langle b_{i}, c_{i}\right\rangle\right) \\
& =\bar{h}(0)=0 . \quad \text { This completes the proof. }
\end{aligned}
$$


Higher $R$-derivations of special subrings of matrix rings

LEMmA 9.3. If in $C_{1}(\Gamma)$ the equality (*) holds then in the ring $T(\Gamma)$ we have

$$
\sum_{i=1}^{k} z_{i}\left\langle a_{i}, b_{i}\right\rangle\left\langle b_{i}, c_{i}\right\rangle\left\langle a_{i}, c_{i}\right\rangle=0 \text {. }
$$

Proof. From Lemma 9.1, for $n=3$, we get

$$
\begin{aligned}
0 & =\sum_{i=1}^{k} z_{i}\left(\left\langle a_{i}, b_{i}\right\rangle^{2}\left\langle b_{i}, c_{i}\right\rangle+\left\langle a_{i}, b_{i}\right\rangle\left\langle b_{i}, c_{i}\right\rangle^{2}\right) \\
& =\sum_{i=1}^{k} z_{i}\left\langle a_{i}, b_{i}\right\rangle\left\langle b_{i}, c_{i}\right\rangle\left(\left\langle a_{i}, b_{i}\right\rangle+\left\langle b_{i}, c_{i}\right\rangle\right) \\
& =\sum_{i=1}^{k} z_{i}\left\langle a_{i}, b_{i}\right\rangle\left\langle b_{i}, c_{i}\right\rangle\left\langle a_{i}, c_{i}\right\rangle .
\end{aligned}
$$

THEOREM 9.4. Every graph $\Gamma$ is a 2-graph over an arbitrary ring $R$.

Proof. Let $f \in T M_{2}\left(\rho^{\prime}, R\right)$ and suppose that in $C_{1}(\Gamma)$ holds $(*)$. Consider the group homomorphism $\varphi: C_{1}(\Gamma) \rightarrow Z(R)$ such that

for all $a \rho^{\prime} b$.

$$
\varphi(a, b)=f_{1}(a, b) f_{2}(a, b)
$$

Then we have

$$
\begin{aligned}
0= & \varphi(0) \\
= & \sum_{i=1}^{k} z_{i}\left(\varphi\left(a_{i}, c_{i}\right)-\varphi\left(a_{i}, b_{i}\right)-\varphi\left(b_{i}, c_{i}\right)\right) \\
= & \sum_{i=1}^{k} z_{i}\left[( f _ { 1 } ( a _ { i } , b _ { i } ) + f _ { 1 } ( b _ { i } , c _ { i } ) ) \left(f_{2}\left(a_{i}, b_{i}\right)+f_{2}\left(b_{i}, c_{i}\right)\right.\right. \\
& \left.\left.+f_{1}\left(a_{i}, b_{i}\right) f_{1}\left(b_{i}, c_{i}\right)\right)-f_{1}\left(a_{i}, b_{i}\right) f_{2}\left(b_{i}, c_{i}\right)\right] \\
= & \sum_{i=1}^{k} z_{i}\left[f_{2}\left(a_{i}, b_{i}\right) f_{1}\left(b_{i}, c_{i}\right)+f_{1}\left(a_{i}, b_{i}\right) f_{2}\left(b_{i}, c_{i}\right)\right] \\
& +\sum_{i=1}^{k} z_{i} f_{1}\left(a_{i}, b_{i}\right) f_{1}\left(b_{i}, c_{i}\right) f_{1}\left(a_{i}, c_{i}\right) .
\end{aligned}
$$

Since, by Lemma 9.3,

$$
\sum_{i=1}^{k} z_{i} f_{1}\left(a_{i}, b_{i}\right) f_{1}\left(b_{i}, c_{i}\right) f_{1}\left(a_{i}, c_{i}\right)=0
$$

then

$$
\sum_{i=1}^{k} z_{i}\left[f_{2}\left(a_{i}, b_{i}\right) f_{1}\left(b_{i}, c_{i}\right)+f_{1}\left(a_{i}, b_{i}\right) f_{2}\left(b_{i}, c_{i}\right)\right]=0 .
$$

This completes the proof.

Using a similar method we can prove the following 
THEOREM 9.5. Let $\Gamma$ be a graph and $R$ be a ring.

a) If $R$ is 2-torsion-free then $\Gamma$ is a 3-graph over $R$,

b) $\Gamma$ is a 4-graph over $R$,

c) If $R$ is 6-torsion-free then $\Gamma$ is a 5-graph over $R$,

d) $\Gamma$ is a 6-graph.

Using the above theorems and arguments from the proof of Theorem 8.6 we obtain

THEORREM 9.6. Let $P$ be a special subring of $M_{n}(R)$ with the relation $\rho$. Assume that the homology group $H(\Gamma(\rho))$ is free abelian. Then

(1) Every $R$-derivation of order $s<3$ of $P$ is 3-integrable.

(2) If $R$ is 2-torsion-free then every $R$-derivation of order $s<5$ of $P$ is 5 integrable.

(3) If $R$ is 3 !-torsion-free then every $R$-derivation of order $s<7$ of $P$ is 7 integrable.

We end this paper with the following open problems:

1). Let $\Gamma=\left(I_{n}, \rho\right)$ be a fixed graph (i.e. $\rho$ is a partial ordering relation on $\left.I_{n}\right)$ and let $s<s^{\prime}$. Suppose that for every $R$ any $R$-derivation of order $s$ of $M_{n}(R)_{\rho}$ is $s^{\prime}$-integrable. Is $H_{1}(\Gamma)$ a free group?

2). Find numbers $n, s$, a ring $R$, and a partial order $\rho$ on $I_{n}$ such that the graph $\Gamma=\left(I_{n}, \rho\right)$ is not $s$-graph over $R$.

3). Is every graph a 3-graph over an arbitrary ring?

\section{References}

[1] Abdeljaouad, M., Note on the automorphisms and derivations of a quasi-matrix algebra. Scient. Papers College Gen. Ed. Univ. Tokyo, 21 (1971), 11-17.

[2] Burkow, W.D., Derivations of generalized quasi-matrix rings(Russian). Mat. Zametki, 24 (1978), 111-122.

[3] Hilton, P.J. and Wylies, S., Homology Theory, Cambridge, 1960.

[4] Matsumura, H., Integrable derivations. Nagoya Math. J., 87 (1982), 227-245.

[5] Miller, J. B., Homomorphisms, higher derivations, and derivations of associative algebras. Acta Sci. Math., 28 (1967), 221-232.

[6] Mürase, I., On the derivations of a quasi-matrix algebra. Scient. Papers College Gen. Ed. Univ. Tokyo, 14 (1964), 157-164.

[7] Nowicki, A., Derivations of special subrings of matrix rings and regular graphs, Tsukuba J. Math., 7 (1983), 281-297.

[8] - Inner derivations of higher orders, Tsukuba J. Math., 8 (1984), 219-225.

[9] Ribenboim, P., Algebraic theory of higher-order derivations. Transactions of the Royal Society of Canada, ser. IV, 7 (1969), 179-187. 
[10] - Higher derivations of rings I. Rev. Roum. Math. Pures Appl., 16 (1971), $77-110$.

[11] - Higher derivations of rings II. Rev. Roum. Math. Pures Appl., 16 (1971), 245-272.

Institute of Mathematics,

N. Copernicous University, Torun 87-100,

ul. Chopina 12/18, Poland. 